\title{
A Single Platinum Microelectrode for Identifying Soft Drink Samples
}

\author{
Lígia Bueno $^{1}$ and Thiago R. L. C. Paixão ${ }^{1,2}$ \\ ${ }^{1}$ Centro de Ciências Naturais e Humanas, Universidade Federal do ABC, Santo André, SP, 09210-170, Brazil \\ ${ }^{2}$ Instituto de Química, Universidade de São Paulo, São Paulo, SP, 05508-900, Brazil \\ Correspondence should be addressed to Thiago R. L. C. Paixão, trlcp@iq.usp.br
}

Received 25 September 2011; Revised 1 November 2011; Accepted 1 November 2011

Academic Editor: Rubin Gulaboski

Copyright (C) 2012 L. Bueno and T. R. L. C. Paixão. This is an open access article distributed under the Creative Commons Attribution License, which permits unrestricted use, distribution, and reproduction in any medium, provided the original work is properly cited.

\begin{abstract}
Cyclic voltammograms recorded with a single platinum microelectrode were used along with a non-supervised pattern recognition, namely, Principal Component Analysis, to conduct a qualitative analysis of sixteen different brands of carbonated soft drinks (Kuat, Soda Antarctica, H2OH!, Sprite 2.0, Guarana Antarctica, Guarana Antarctica Zero, Coca-Cola, Coca-Cola Zero, Coca-Cola Plus, Pepsi, Pepsi Light, Pepsi Twist, Pepsi Twist Light, Pepsi Twist 3, Schin Cola, and Classic Dillar's). In this analysis, soft drink samples were not subjected to pre-treatment. Good differentiation among all the analysed soft drinks was achieved using the voltammetric data. An analysis of the loading plots shows that the potentials of $-0.65 \mathrm{~V},-0.4 \mathrm{~V}, 0.4 \mathrm{~V}$, and $0.750 \mathrm{~V}$ facilitated the discrimination process. The electrochemical processes related to this potential are the reduction of hydrogen ions and inhibition of the platinum oxidation by the caffeine adsorption on the electrode surface. Additionally, the single platinum microelectrode was useful for the quality control of the soft drink samples, as it helped to identify the time at which the beverage was opened.
\end{abstract}

\section{Introduction}

The food market is one of the fastest growing economic sectors in the world. The development of new smart devices for identifying adulteration, detecting alteration of organoleptic properties, and consistently implementing storage processes [1-4] could enhance the value of food products and thereby prevent product losses. For some food products, authenticity is also very important, especially when companies that distribute them seek market exclusivity. Thus, the development of devices that could be used to identify the origins of food products would be very useful. Much work has already been carried out on the quantification of individual compounds in food samples $[5,6]$ using expensive and complex techniques. In addition, the use of techniques to differentiate between food products based on fingerprints of food samples has been increasing over the last ten years $[1,4,7-10]$.

Array of nonspecific, low-selective, chemical sensors with an appropriate method of pattern recognition (called electronic tongue) [11] can make the analysis of foods faster and easier. An electronic tongue does not require the sample to be treated beforehand and does not destroy the sample during the analysis, as is the case with spectrometric techniques $[5,12]$. In addition, its operation is based on the global selectivity concept. Therefore, it does not necessarily quantify the analytes in a sample; rather, it collects fingerprint data of the sample, such as UV-Vis spectra or voltammetric or capacitance versus frequency behaviour [13-16].

Even though the use of intelligent devices in the liquid phase is a relatively new practice, a variety of samples have been analysed [3, 10, 11, 17-20]. Electronic tongues facilitate differentiation within short times, result in low costs, and have nondestructive characteristics. Therefore, they have attracted the attention of companies in the food industry, as well as researchers in the pharmaceutical and environmental sciences.

The market share of soft drink consumption was $46.8 \%$ for the nonalcoholic drinks industry in 2005 [21], and the total market value of soft drinks reached $\$ 307.2$ billion in 2004 , with a market value forecast of $\$ 367.1$ billion in 2009. Clearly, the soft drink market is lucrative and has the potential for high profits. Therefore, this study is aimed at 
developing a low-cost analytical method that can be applied in the field to discriminate the origin of these nonalcoholic drinks using voltammetric data recorded by employing only a single working electrode (platinum microelectrode) along with unsupervised pattern recognition instead of an array of sensors (electronic tongue). To the authors' knowledge, only two prior studies have focused on differentiation between carbonated soft drinks using an electronic tongue with colorimetric detection [22] and 12 different highly cross-sensitive potentiometric sensors [23].

\section{Experimental Section}

2.1. Chemicals and Materials. Sulphuric acid was purchased from Merck (Darmstadt, Germany) and was used without further purification. Deionised water was produced using a water purification system (Direct-Q 5 Ultrapure Water Systems, Millipore, USA).

2.2. Electrodes and Instrumentation. A $\mu$ Autolab (Eco Chemie, Netherlands) with GPES 4.8 software was used to take electrochemical measurements. In experiments that were carried out using a platinum disk working electrode $(d=$ $3 \mathrm{~mm}$ ), a homemade $\mathrm{Ag} / \mathrm{AgCl}$ (saturated $\mathrm{KCl}$ ) electrode [24] and platinum coil counter electrode were used. Platinum microelectrodes with a diameter of $25 \mu \mathrm{m}$ were constructed using a pipette puller machine according to a technique reported in the literature [25]. The working electrode surface was polished with alumina $(0.3 \mu \mathrm{m})$ and then washed thoroughly with deionised water before taking electrochemical measurements.

2.3. Soft Drink Samples. Sixteen different brands of soft drinks (Kuat, Soda Antarctica, H2OH!, Sprite 2.0, Guaraná Antarctica, Guaraná Antarctica Zero, Coca-Cola Classic, Coca-Cola Zero, Coca-Cola Plus, Pepsi Classic, Pepsi Light, Pepsi Twist, Pepsi Twist Light, Pepsi Twist 3, Schin Cola, and Classic Dillar's) purchased from different supermarkets in Santo André, Brazil, were used in this study. The measurements were performed directly on the soft drink samples without the addition of other chemicals.

2.4. Chemometrics. Principal component analysis (PCA) and hierarchical cluster analysis (HCA) were performed using Statistica 10.0 (StatSoft Inc., USA). The analyses were carried out using the current values recorded without any preprocessing of the input. A range of potentials from 0.8 to $-0.8 \mathrm{~V}$ were recorded in triplicate for each independent sample using previously polished working electrodes.

\section{Results and Discussion}

Initially, in order to discriminate between cola soft drinks, cyclic voltammograms were recorded using a platinum working macroelectrode that had been placed directly in the untreated beverages. The voltammograms (not shown) showed that the $\mathrm{CO}_{2}$ present in each beverage interfered with the measurements, resulting in very noisy and irreproducible voltammetric behaviour. Therefore, it was difficult

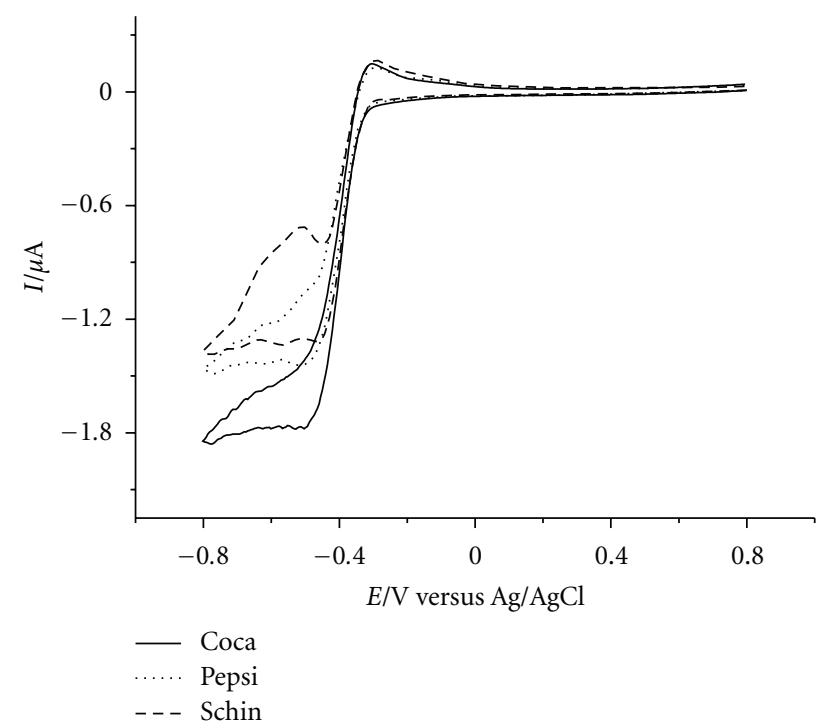

Figure 1: Cyclic voltammograms recorded using a platinum microelectrode $(d=25 \mu \mathrm{m})$ placed directly in the soft drink samples: Pepsi (dotted line), Coca-Cola (solid line), and Schin Cola (short dashed line). Scan rate: $50 \mathrm{mV} \mathrm{s}^{-1}$.

to differentiate between the soft drinks. In order to avoid such problems and due to convection generated by the $\mathrm{CO}_{2}$ bubbles being neglected at electrodes of micrometric dimensions [26], a platinum microelectrode $(d=25 \mu \mathrm{m})$ was used as the working electrode to take voltammetric measurements. The voltammograms recorded directly using the beverages clearly show that the voltammetric behaviour for each brand of soft drink was different (Figure 1). The main difference is related to the reduction in the region of $-0.4 \mathrm{~V}$ versus $\mathrm{Ag} / \mathrm{AgCl}$ (Figure 1), which is associated with the reduction of hydrogen ions [27] in different soft drinks.

After verifying the possibility of extracting information on the reduction of hydrogen ions at a platinum microelectrode in order to differentiate between soft drinks, studies aimed at differentiating between samples according to the ingredient and/or type of soft drink were initiated. Initially, tests were performed with cola soft drinks comprising three different brands in their original versions: Coca-Cola, Pepsi, and Schin. For the chemometric treatments, the current values were used as inputs for the PCA. To this end, a primary matrix composed of 9 rows and 656 columns was analysed using Statistica software. Figure 2 shows the score plot for the voltammetric measurements obtained directly from the cola soft drink samples. By analysing the data presented in Figure 2, a clear distinction can be made between the CocaCola, Pepsi, and Schin beverages using two first principal components with $85.85 \%$ of the total information collected by the platinum microelectrodes.

The discrimination that is displayed in Figure 2 can also be explained by using the voltammograms of each brand of beverage (Figure 1). Here, small differences were observed between the soft drink profiles, especially for the signal relative to the reduction of hydrogen ions. The different values of the limiting current for proton reduction in samples 


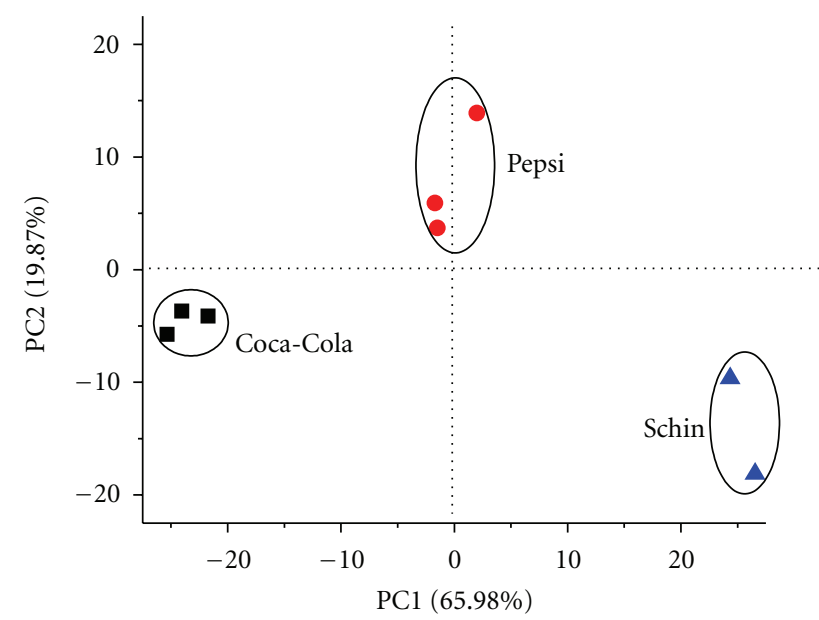

Figure 2: A PCA score biplot for cola soft drinks (Pepsi, CocaCola, and Schin Cola). The biplot was obtained using current values recorded with a platinum microelectrode. Number of replicates per sample: 3 .

could be related to the interference of various substance in the reduction process of $\mathrm{H}^{+}$, which could result in the differentiation of sample with the same $\mathrm{pH}$ values of each drink (Coca-Cola Classic $(\mathrm{pH}=2.50)$ and Pepsi $(\mathrm{pH}=2.47)$ [22]), validate the idea that each type of soft drink has a unique fingerprint [28].

Additionally, the loading plots in Figure 2 (not shown) were analysed to determine the weights of the different parts of the voltammograms (current values measured at different potentials) in relation to the extent of their influence on the direction of the principal components considered in the differentiation process. According to this analysis, the currents obtained for the potentials $-0.65 \mathrm{~V},-0.4 \mathrm{~V}, 0.4 \mathrm{~V}$, and $0.750 \mathrm{~V}$ facilitated the differentiation shown in Figure 2. This supports the assumption that differentiation can be achieved based on the current for the reduction of the hydrogen ions at the potentials $-0.65 \mathrm{~V}$ and $-0.4 \mathrm{~V}$. The other two potentials are discussed at a subsequent point in this article.

After confirming that it is possible to discriminate between cola soft drinks of different brands, the possibility of differentiating between different types of soft drinks of the same or different brands was evaluated. The brands and types studied were Pepsi Classic, Pepsi Light, Pepsi Light Twist, Pepsi Twist, Coca-Cola Classic, Coca-Cola Zero, and Coca-Cola Plus. A similar procedure to the one reported for the results in Figure 2 was employed. The score plot obtained is shown in Figure 3. In this case, the voltammetric data recorded with a single platinum microelectrode with unsupervised pattern recognition was able to differentiate between the brands Coca-Cola and Pepsi, which are represented on opposite sides of the score plot. This emphasizes the possibility of differentiating between samples using the proposed method. In order to show the degree of similarity of the PCA score plots more quantitatively, HCA was performed using the data's full dimensionality of the data reported in the Figure 3 (results not shown). No misclassifications ( $0 \%$ error rate) were found

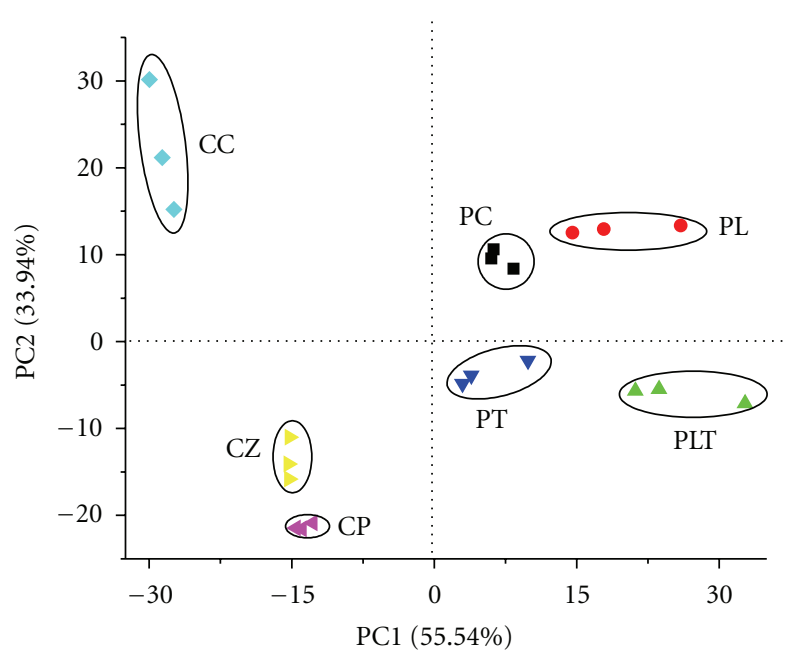

FIGURE 3: A PCA score biplot for different brands of cola soft drinks. The biplot was obtained using current values recorded with a platinum microelectrode. Number of replicates per sample: 3 . Abbreviations: Pepsi Classic (PC), Pepsi Light (PL), Pepsi Light Twist (PLT), Pepsi Twist (PT), Coca-Cola Classic (CC), Coca-Cola Zero (CZ), and Coca-Cola Plus (CP).

and the discrimination was consistent with the PCA results (Figure 3).

Once the possibility of differentiating between the cola soft drink samples was demonstrated, new samples were tested. They were Kuat, Soda Antarctica, H2OH!, Sprite 2.0, Guaraná Antarctica, Guaraná Antarctica Zero, CocaCola, Coca-Cola Zero, Pepsi, Pepsi Light, Pepsi Twist, Pepsi Twist Light, Pepsi Twist 3, Schin Cola, and Classic Dillar's. A similar procedure to the one for producing the data in Figures 2 and 3 was employed (Figure 4). Separation of the brands was notable, and proved that our device can be used to differentiate between samples with and without cola in their formulation. Samples containing cola were opposite to those that did not contain cola, which shows that the separation was not a result of only the $\mathrm{pH}$ values of beverages. This result corroborates the result obtained using the device proposed by Zhang and Suslick [22], which was based on colorimetric device. Applying an HCA model (not shown) there is only a single case of misclassification in the 45 samples analysed (one Guaraná Antarctica sample did not similar to the other 2 samples of the same soft drink), that is, a misclassification rate of $2.2 \%$.

To understand which species were responsible for the differences between soft drinks and why the currents recorded at $0.4 \mathrm{~V}$ and $0.750 \mathrm{~V}$ were important for differentiation, new studies were performed. In particular, the studies were performed to verify whether caffeine content, which differs between cola and non-cola soft drinks, facilitates the differentiation. Caffeine interacts with the surface of the platinum electrode [29]. Hence, cyclic voltammograms were recorded in the presence and absence of caffeine (Figure 5). Blocking of the platinum surface by adsorbed caffeine molecules was observed (Figure 5). In other words, there was a reduction in the electroactive platinum area and consequent reduction 


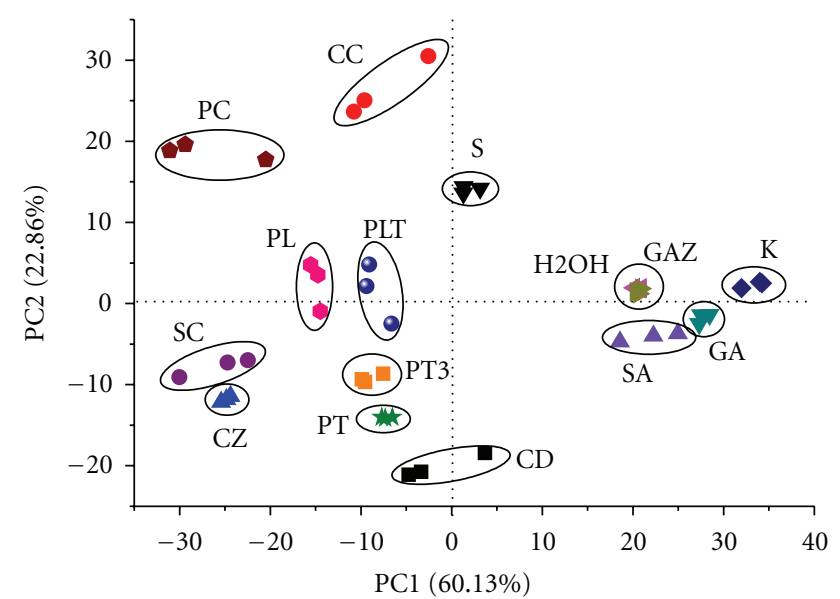

Figure 4: A PCA score biplot for 15 different brands of soft drinks. The biplot was obtained using current values recorded with a platinum microelectrode. Number of replicates per sample: 3 . Abbreviations: Kuat (K), Soda Antarctica (SA), H2OH! (H2OH), Sprite 2.0 (S), Guaraná Antarctica (GA), Guaraná Antarctica Zero (GAZ), Coca-Cola Classic (CC), Coca-Cola Zero (CZ), Pepsi Classic (PC), Pepsi Light (PL), Pepsi Twist (PT), Pepsi Twist Light (PTL), Pepsi Twist 3 (PT3), Schin Cola (SC), and Classic Dillar's (CD).

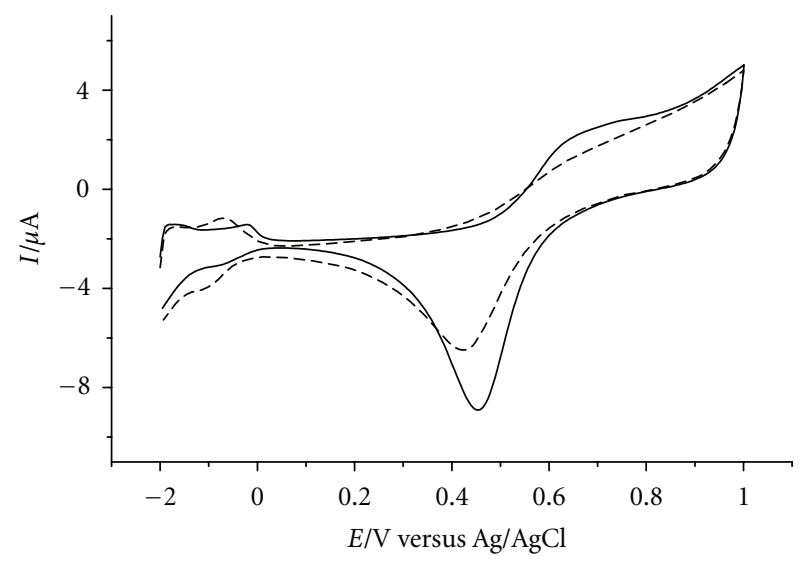

FIGURE 5: Cyclic voltammograms recorded using a platinum working electrode in $0.01 \mathrm{~mol} \mathrm{~L}^{-1} \mathrm{H}_{2} \mathrm{SO}_{4}$ solution before (solid line) and after the addition of caffeine (dashed line, final concentration $=0.25$ mmol L $\left.{ }^{-1}\right)$. Scan rate: $50 \mathrm{mV} \mathrm{s}^{-1}$.

in the extent of electrochemical processes that occurred with platinum in acid at $0.4 \mathrm{~V}$ and $0.750 \mathrm{~V}$.

Considering the decrease in current values for the platinum electrochemical process as a consequence of the blocking effect of caffeine, it can be concluded that the differentiation between soft drinks was also possibly due to the adsorption of caffeine onto the electrode surface. This decrease in the area of electroactive platinum/current signal of platinum process is proportional to the amount of caffeine in the beverage. The acidity of the beverage also helps to differentiate between beverages, since the electrode is able to extract information on the reduction of hydrogen ions.

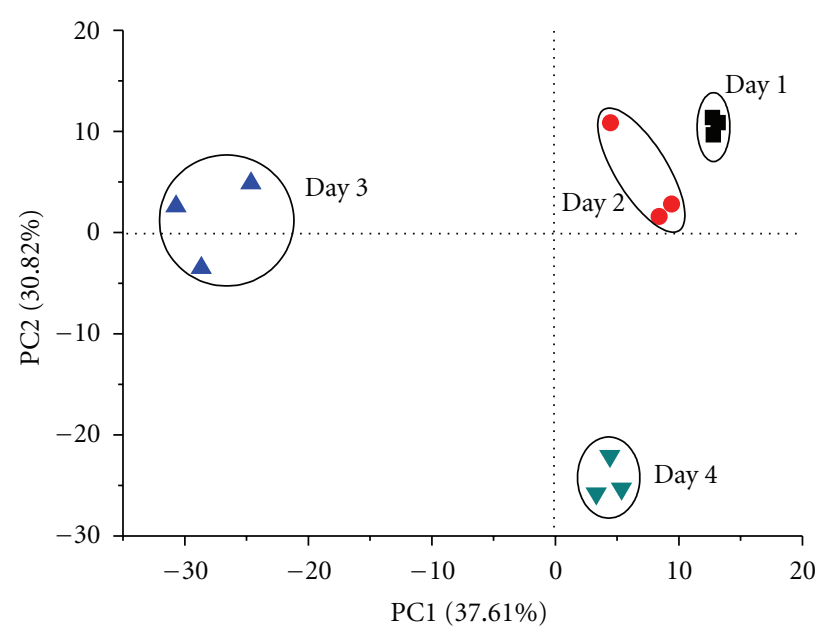

Figure 6: PCA score biplot for opened Coca-Cola samples. The measurements are for day 1 (just opened), and 2, 3, and 4 days after opening. The biplot was obtained using current values recorded with a platinum microelectrode. Number of replicates per sample: 3.

This inference is in agreement with the results of a previous study [22], $\mathrm{pH}$ was not the only variable responsible for the discrimination.

Lastly, another method can be used in conjunction with our proposed method for quality control of soft drink production. Three voltammograms were recorded for CocaCola and Pepsi samples over four days after bottles of each soft drink had been opened. Between measurements, the bottles were closed and stored at room temperature. The current values recorded were used as input data for PCA. Figure 6 shows the score plot for the experiment performed with Coca-Cola. All triplicate measurements for each day were reasonably well clustered and separated from the other measurements taken on other days. The same behaviour was observed for the Pepsi samples (not shown). The explanation for this behaviour is that the loss of $\mathrm{CO}_{2}$ over time resulted in different $\mathrm{pH}$ values for the soft drink samples. As the electrode could differentiate between the samples according to their proton content, it could be used for soft drink quality assurance.

\section{Conclusions}

A single platinum microelectrode can be used as a device to differentiate between soft drink samples of discriminate compositions and origins. The input data for the differentiation process using unsupervised pattern recognition method are current values recorded between 0.8 and $-0.8 \mathrm{~V}$ versus $\mathrm{Ag} / \mathrm{AgCl}$ using a platinum microelectrode. The different locations of the data in terms of the loading plots associated with the sensor show that the $\mathrm{pH}$ values and caffeine content present in the beverages are responsible for the differences between the samples. A database containing information on a large variety of soft drinks that were analysed previously suggests that our method may be useful to classify the brand type, origin, and quality of a soft drink. 


\section{Acknowledgments}

The authors are grateful to FAPESP (Fundação de Amparo à Pesquisa do Estado de São Paulo; Grant number 2009/078591), CAPES, and CNPq (Conselho Nacional de Desenvolvimento Científico e Tecnológico) for financial support and, once again, to CAPES for the fellowship awarded to L. Bueno.

\section{References}

[1] L. Escuder-Gilabert and M. Peris, "Review: highlights in recent applications of electronic tongues in food analysis," Analytica Chimica Acta, vol. 665, no. 1, pp. 15-25, 2010.

[2] P. Ciosek and W. Wróblewski, "Potentiometric electronic tongues for foodstuff and biosample recognition-an overview," Sensors, vol. 11, no. 5, pp. 4688-4701, 2011.

[3] J. Gallardo, S. Alegret, and M. Del Valle, "Application of a potentiometric electronic tongue as a classification tool in food analysis," Talanta, vol. 66, no. 5, pp. 1303-1309, 2005.

[4] A. Legin, A. Rudnitskaya, Y. G. Vlasov, C. Di Natale, F. Davide, and A. D'Amico, "Tasting of beverages using an electronic tongue," Sensors and Actuators B, vol. 44, no. 1-3, pp. 291-296, 1997.

[5] O. L. A. D. Zucchi, S. Moreira, M. J. Salvador, and L. L. Santos, "Multielement analysis of soft drinks by x-ray fluorescence spectrometry," Journal of Agricultural and Food Chemistry, vol. 53, no. 20, pp. 7863-7869, 2005.

[6] M. S. García-Falcón and J. Simal-Gándara, "Determination of food dyes in soft drinks containing natural pigments by liquid chromatography with minimal clean-up," Food Control, vol. 16, no. 3, pp. 293-297, 2005.

[7] Q. Chen, J. Zhao, and S. Vittayapadung, "Identification of the green tea grade level using electronic tongue and pattern recognition," Food Research International, vol. 41, no. 5, pp. 500504,2008

[8] P. Ciosek and W. Wróblewski, "Miniaturized electronic tongue with an integrated reference microelectrode for the recognition of milk samples," Talanta, vol. 76, no. 3, pp. 548-556, 2008.

[9] A. L. A. Rudnitskaya, B. Seleznev, and Y. Vlasov, "Recognition of liquid and flesh food using an 'electronic tongue'” International Journal of Food Science and Technology, vol. 37, no. 4, pp. 375-385, 2002.

[10] E. A. Baldwin, J. Bai, A. Plotto, and S. Dea, "Electronic noses and tongues: applications for the food and pharmaceutical industries," Sensors, vol. 11, no. 5, pp. 4744-4766, 2011.

[11] Y. Vlasov, A. Legin, A. Rudnitskaya, C. Di Natale, and A. D'Amico, "Nonspecific sensor arrays ("electronic tongue") for chemical analysis of liquids: (IUPAC technical report)," Pure and Applied Chemistry, vol. 77, no. 11, pp. 1965-1983, 2005.

[12] A. Stój, "Methods of detecting adulteration of wines," Zywnosc-Nauka Technologia Jakosc, vol. 18, no. 2, pp. 17-26, 2011.

[13] M. Casale, P. Oliveri, C. Armanino, S. Lanteri, and M. Forina, "NIR and UV-vis spectroscopy, artificial nose and tongue: comparison of four fingerprinting techniques for the characterisation of Italian red wines," Analytica Chimica Acta, vol. 668, no. 2, pp. 143-148, 2010.

[14] E. S. Medeiros, R. Gregório Jr., R. A. Martinez, and L. H. C. Mattoso, "A taste sensor array based on polyaniline nanofibers for orange juice quality assessment," Sensor Letters, vol. 7, no. 1, pp. 24-30, 2009.
[15] R. H. Labrador, J. Olsson, F. Winquist, R. Martínez-Máñez, and J. Soto, "Determination of bisulfites in wines with an electronic tongue based on pulse voltammetry," Electroanalysis, vol. 21, no. 3-5, pp. 612-617, 2009.

[16] F. Winquist, R. Bjorklund, C. Krantz-Rülcker, I. Lundström, K. Östergren, and T. Skoglund, "An electronic tongue in the dairy industry," Sensors and Actuators B, vol. 111-112, pp. 299-304, 2005.

[17] W. Novakowski, M. Bertotti, and T. R. L. C. Paixão, "Use of copper and gold electrodes as sensitive elements for fabrication of an electronic tongue: discrimination of wines and whiskies," Microchemical Journal, vol. 99, no. 1, pp. 145-151, 2011.

[18] L. M. I. Codinachs, J. P. Kloock, M. J. Schöning et al., "Electronic integrated multisensor tongue applied to grape juice and wine analysis," Analyst, vol. 133, no. 10, pp. 1440-1448, 2008.

[19] F. Winquist, C. Krantz-Rülcker, P. Wide, and I. Lundström, "Monitoring of freshness of milk by an electronic tongue on the basis of voltammetry," Measurement Science and Technology, vol. 9, no. 12, pp. 1937-1946, 1998.

[20] T. R. L. C. Paixão and M. Bertotti, "Fabrication of disposable voltammetric electronic tongues by using Prussian Blue films electrodeposited onto CD-R gold surfaces and recognition of milk adulteration," Sensors and Actuators B, vol. 137, no. 1, pp. 266-273, 2009.

[21] Datamonitor, "Global Soft Drinks: Industry Profile," New York, NY, USA. Reference Code: 0199-0802, 2005.

[22] C. Zhang and K. S. Suslick, "Colorimetric sensor array for soft drink analysis," Journal of Agricultural and Food Chemistry, vol. 55, no. 2, pp. 237-242, 2007.

[23] L. Lvova, S. S. Kim, A. Legin et al., "All-solid-state electronic tongue and its application for beverage analysis," Analytica Chimica Acta, vol. 468, no. 2, pp. 303-314, 2002.

[24] J. J. Pedrotti, L. Angnes, and I. G. R. Gutz, "Miniaturized reference electrodes with microporous polymer junctions," Electroanalysis, vol. 8, no. 7, pp. 673-675, 1996.

[25] P. N. Bartlett and D. J. Caruana, "Electrochemical immobilization of enzymes part V. $*$ Microelectrodes for the detection of glucose based on glucose oxidase immobilized in a poly(phenol) film," The Analyst, vol. 117, no. 8, pp. 1287-1292, 1992.

[26] C. Amatore, C. Pebay, L. Thouin, A. Wang, and J. S. Warkocz, "Difference between ultramicroelectrodes and microelectrodes: influence of natural convection," Analytical Chemistry, vol. 82, no. 16, pp. 6933-6939, 2010.

[27] Z. Yoshida, "Preparation of an ideal thin mercury film electrode and its electrochemical property," Bulletin of the Chemical Society of Japan, vol. 54, no. 2, pp. 562-567, 1981.

[28] A. Riul, D. S. Dos Santos, K. Wohnrath et al., "Artificial taste sensor: efficient combination of sensors made from LangmuirBlodgett films of conducting polymers and a ruthenium complex and self-assembled films of an azobenzene-containing polymer," Langmuir, vol. 18, no. 1, pp. 239-245, 2002.

[29] P. Saba, W. A. Brown, and S. Omanovic, "Interactive behavior of caffeine at a platinum electrode surface," Materials Chemistry and Physics, vol. 100, no. 2-3, pp. 285-291, 2006. 


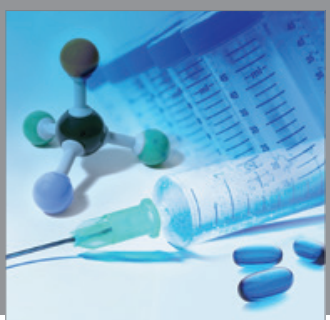

International Journal of

Medicinal Chemistry

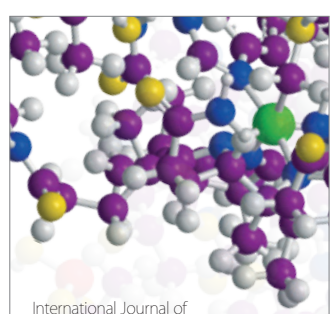

Carbohydrate Chemistry

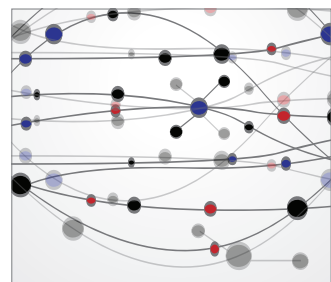

The Scientific World Journal
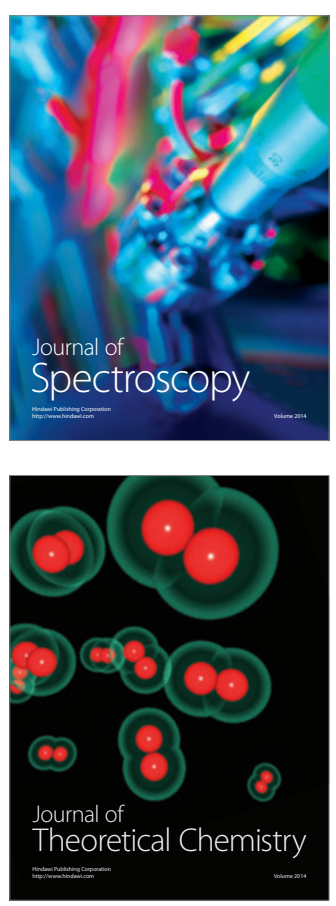
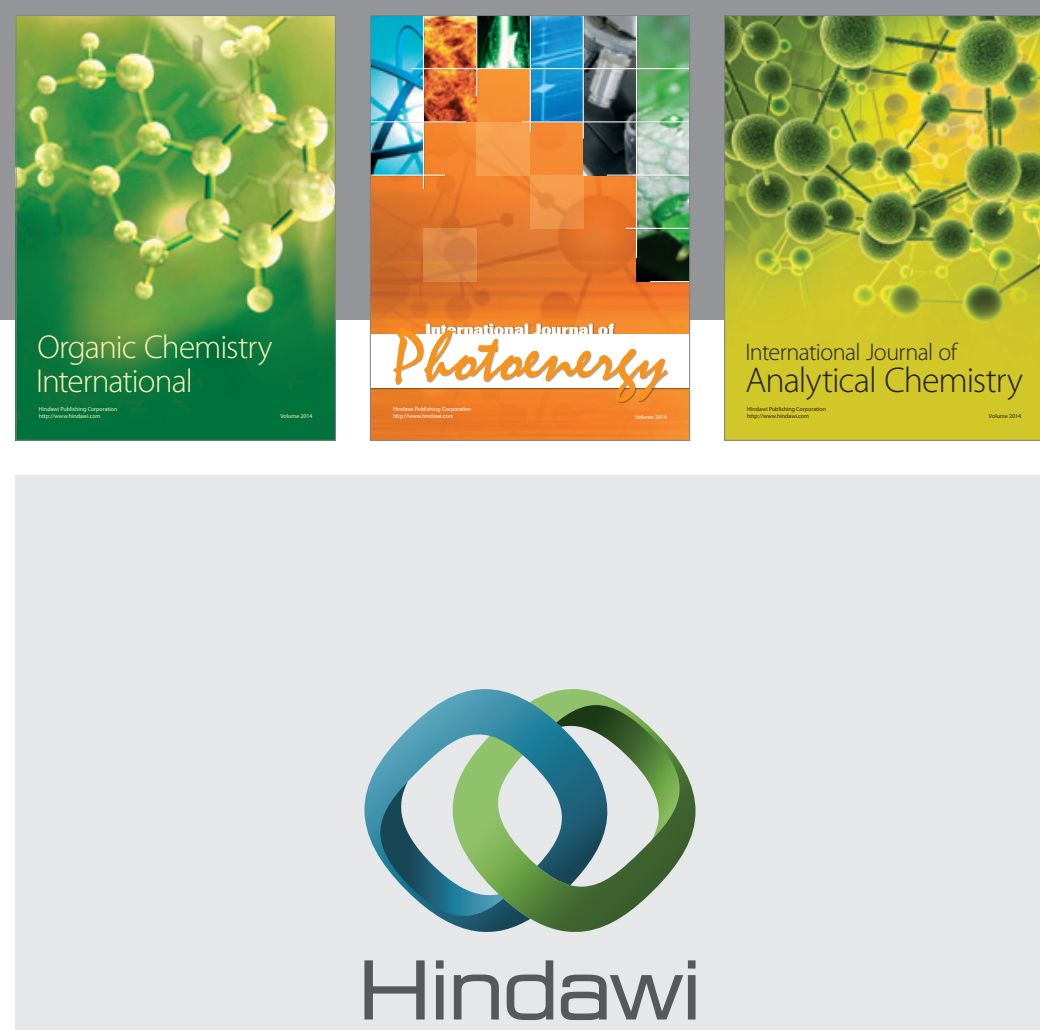

Submit your manuscripts at

http://www.hindawi.com
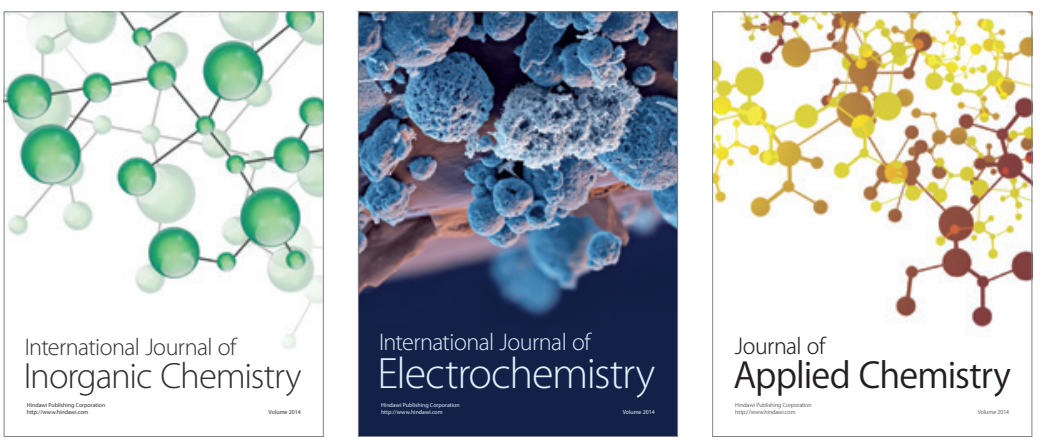

Journal of

Applied Chemistry
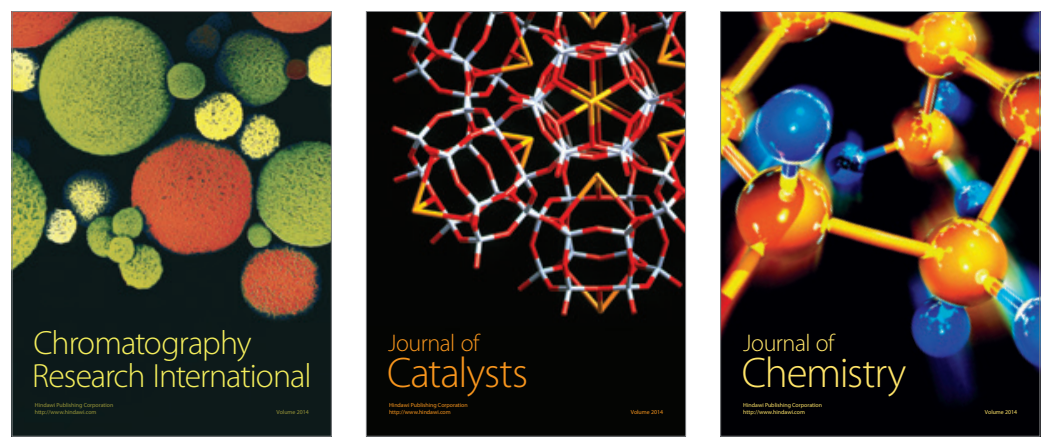
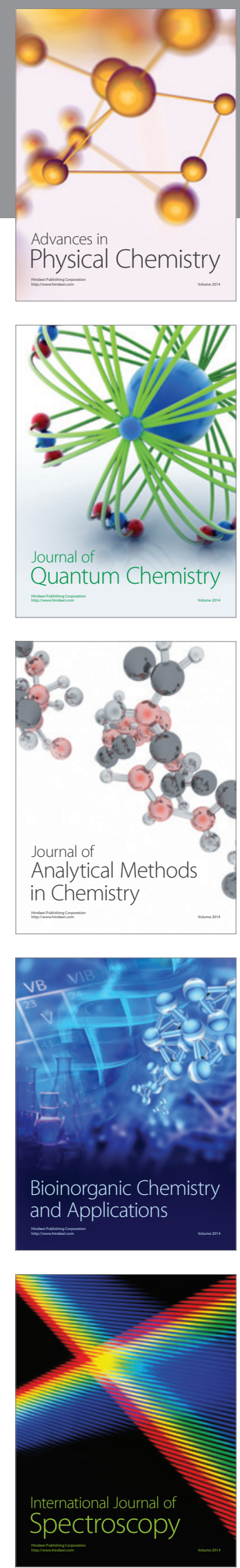